\title{
COMPUTING THE DAMPING OF SUBSYNCHRONOUS OSCILLATIONS DUE TO A THYRISTOR CONTROLLED SERIES CAPACITOR
}

\author{
Rajesh Rajaraman \\ Student Member
}

\author{
Ian Dobson \\ Member
}

Electrical and Computer Engineering Dept.

University of Wisconsin, Madison, WI 53706
Yihchih Shern

Student Member

\begin{abstract}
We extend eigenvalue analysis of subsynchronous resonance (SSR) to include thyristor switchings. The eigenvalue computation takes account of the time variations and nonlinearities of both the thyristor switchings and the generator. An example is presented using the IEEE first benchmark SSR model. The paper contributes an accurate method to compute the damping of the SSR modes with a TCSC.
\end{abstract}

Keywords: flexible $\mathrm{AC}$ transmission system, thyristor controlled series capacitor, subsynchronous resonance, Poincaré map, small signal stability, eigenvalue.

\section{Introduction}

Subsynchronous resonance and the associated turbine-generator torsional interactions are a major constraint for many series compensated transmission systems. Series capacitor compensation of long transmission lines increases their power transfer, but may inadvertently increase the potential for subsynchronous resonance (SSR). Such torsional interactions are an instability in which large subsynchronous torques can cause extensive damage to turbine-generator shafts [1]. Flexible AC transmission systems such as the Thyristor Controlled Series Capacitor (TCSC) offer the possibility of power flow control and suppression of SSR instabilities through controlled series compensation.

Analytical tools for the study of SSR problems without thyristor controlled devices are well established and include frequency scan methods, eigenvalue analysis, and time domain simulation. These approaches are being developed to study SSR problems with thyristor controlled devices, but there are some problems in accurately representing the thyristor switchings. A recent IEEE committee reported [2] that one of the shortcomings of eigenvalue analysis was that 'Switching devices, e.g., thyristor valves, are represented by approximated linear transfer functions which neglect the possible impact of switching on the system behavior'.

The papers on the inclusion of the TCSC in SSR

95 SM 403-6 PWRD A paper recommended and approved by the IEEE Transmission and Distribution Committee of the IEEE Power Engineering society for presentation at the 1995 IEEE/PES Summer Meeting, July 23-27, 1995, Portland, OR. Manuscript submitted July 25, 1994; made available for printing April 27, 1995. studies are centered on two field installations, Western Area Power Administration's Kayenta site [3,4], and Bonneville Power Administration's Slatt substation $[5,6]$. The Kayenta system was analyzed with time domain simulation of a detailed model of the AC system, a machine and TCSC with controls [4]. The measured response at a subsynchronous frequency $(35 \mathrm{~Hz})$ showed pure capacitive behavior at small TCSC conduction angles and inductive behavior with resistance for conduction angles above $20^{\circ}$. This implies that a $35 \mathrm{~Hz}$ subsynchronous instability would be eliminated if the TCSC was operated with conduction angles above $20^{\circ}$.

For the Slatt system, the effect of the TCSC was evaluated by measuring the electrical damping torque as a function of machine rotor speed. Analog and digital simulators modeled the AC system, machine and TCSC with controls. In the analog studies, a transfer function was calculated by modulating the synchronous machine rotor speed with white noise. As the conduction interval of the TCSC was increased, the negative electrical damping was significantly reduced at the critical torsional modes [5]. In the digital studies [6], the TCSC was represented by linear transfer functions obtained from time domain simulations. This work also shows the possibility of damping subsynchronous oscillations.

This paper presents a method for computing the eigenvalues and damping of SSR modes when the system includes a switching device. The computation avoids time domain simulations. The paper is organized as follows: Section 2 outlines an eigenvalue analysis method which takes detailed account of the thyristor switchings. Section 3 describes a simple test case based on the IEEE SSR first benchmark model [7]. Sections 4, 5, 6 contain detailed descriptions of the system equations, their linearization and the computation of the eigenvalues and modal dampings. Section 7 shows the eigenvalue analysis of the test case and the damping of SSR by the TCSC. Section 8 supports the eigenvalue results with ElectroMagnetic Transient Program (EMTP) time domain simulations and Section 9 concludes the paper. The Appendix gives the system parameters.

\section{Analytical approach}

Both generators and TCSCs are nonlinear and time varying devices; when included together in a system, they further complicate analysis. For example, if the load is balanced, the generator equations simplify with Park's transformation so that an observer on the generator rotor sees a time invariant system. However, if a TCSC is included, the system is instantaneously unbal- 
anced whenever some thyristor are on and others are off, and the observer on the rotor sees the TCSC as a nasty time varying system.

The thyristor controlled reactors of the TCSC are switched in and out of the circuit in a regular pattern and our analysis reflects this by modifying the system equations accordingly at each switching. The TCSC is nonlinear because the thyristor switch off times depend on the thyristor current and this is accounted for in the analysis.

Our overall approach is to sample the system once every supply cycle to obtain a discrete time system called the Poincaré map [8]. At steady state periodic operation, if one 'strobes' the system once every period, then the samples at each flash of the strobe will be constant, i.e., the Poincaré map will have a fixed point. Moreover, the small signal behavior of the system corresponds exactly to the small signal behavior of the Poincaré map. In particular, the eigenvalues of the Jacobian of the Poincaré map describe the modal damping of the system.

The system equations can be obtained by a judicious combination of the standard techniques of Anderson $[9,10]$ to describe the generator dynamics in 0dq axes and Jalali $[11,12]$ to describe the TCSC taking proper account of the thyristor switchings including synchronization and controls. Steinbuch [13] has also analyzed a similar problem in linearizing a synchronous generator supplying a three phase rectifier. The system is analytically linearized about the steady state $[9,10,11,13]$. The system linearization depends on the steady state periodic operating point and this steady state is computed numerically. The system linearization varies over the supply cycle and is numerically integrated to yield the Jacobian of the Poincaré map. Modal dampings are then computed from the eigenvalues of the Jacobian.

\section{Test case description}

The first benchmark model was created by the IEEE SSR Task Force [7] as a standard test model to facilitate analysis and comparison of calculations of SSR. The electrical part of the system is shown in Fig. 1.

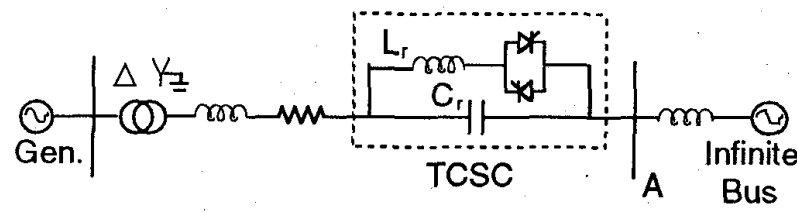

Figure 1. IEEE First Benchmark Model with TCSC.

The turbine-generator shaft is modeled as 6 masses with torsional springs. There are five torsional modes TM1 through TM5 for the mechanical system with respective frequencies $16,20,25,32$ and $47 \mathrm{~Hz}$. The TM4 mode is highly unstable when the series capacitors compensation level is $29 \%$ of the combined transmission and transformer impedances. There is also a rigid body mode TM0 corresponding to power swings of the system.
Each phase of the TCSC consists of a fixed capacitor with a parallel connected thyristor controlled reactor as shown in Fig. 1. The switching element is two oppositely poled thyristors which conduct on alternate half cycles of the supply frequency. The reactor is sized so that the resonance between the capacitor and parallel reactor is below the third harmonic [3]. System parameters are given in the Appendix and [7]. The TCSC changes from capacitative to inductive operation at a conduction angle of $68^{\circ}$.

Our main objective in this paper is to perform exact eigenvalue analysis of the simplest possible case which has the combined difficulties of an electromechanical machine model and thyristor switchings. The addition of the TCSC to the IEEE first SSR benchmark model provides such a test case and the main objective is fulfilled in the detailed analysis in Sections 4 and 5 . Section 7 illustrates the results of the analysis by computing the eigenvalues of the test case. Jalali [11] shows how to linearize TCSC synchronization and a closed loop control. Jalali's equations can be added to those of Sections 4 and 5 to obtain eigenanalysis of the full system. Synchronization and control can have a significant effect on system stability, but here we use a constant firing angle for the TCSC for simplicity.

\section{State Space Equations}

We derive the state space equations for the first SSR benchmark model with the TCSC. All quantities including time are assumed to be in per unit. Some details of the derivation and notation must be found in $[7,9,10]$.

We model the thyristor as an ideal diode with a gate. The thyristor turns on when a firing pulse is applied at the gate, conducts current only in the forward direction, and turns off when the current through the thyristor becomes zero.

One of the possible modes of operation of the three phase TCSC is when one thyristor conducting alternates with no thyristors conducting ( $\sigma<60^{\circ}$ as shown in Fig. 2). Other modes of operation of the three phase TCSC arise when $\sigma>60^{\circ}$. In particular, when $60^{\circ}<\sigma<120^{\circ}$, one thyristor conducting alternates with two thyristors conducting and when $120^{\circ}<\sigma<180^{\circ}$, two thyristors conducting alternates with three thyristors conducting. Here we assume the mode of operation with $\sigma<60^{\circ}$; similar equations can be developed for the other modes.

The current through the conducting thyristor is denoted $i_{r}$. We express all the other stator and transmission line variables in the 0dq axes. The capacitor voltages are thus $\left(v_{0}, v_{d}, v_{q}\right)$ and the line currents $\left(i_{0}, i_{d}, i_{q}\right)$. The infinite bus is a three phase voltage source with line to line voltage $V_{\infty}$ and angle $\alpha$. The synchronous machine field voltage and field current are denoted by $V_{F}$ and $i_{F}$ respectively; $i_{D}, i_{Q}$ and $i_{G}$ are respectively the currents in the d-axis amortisseur, q-axis amortisseur and q-axis deep amortisseur windings. The power angle is denoted 
by $\delta$ and the generator angular speed is denoted by $\omega$. The line capacitance and thyristor controlled reactor inductance are $C_{r}$ and $L_{r}$ respectively; $\mathbf{R}$ and $\mathbf{L}$ are the three phase system resistance and inductance matrices. The electrical system inputs $u_{e}$ are the infinite bus voltage projected onto the rotor dq axes and the field voltage so that

$$
u_{e}=\left(-V_{\infty} \sin (\delta-\alpha), V_{\infty} \cos (\delta-\alpha),-V_{F}\right)^{t}
$$

We first determine the electrical system equations when the thyristor in phase $\mathrm{A}$ is conducting; the method is similar when the thyristor in phase $\mathrm{B}$ or $\mathrm{C}$ is conducting. Then the electrical state vector is

$$
x_{e}=\left(i_{0}, i_{d}, i_{q}, i_{F}, i_{D}, i_{Q}, i_{G}, v_{0}, v_{d}, v_{q}, i_{r}\right)^{t}
$$

and using the techniques similar to [10], the electrical system dynamics are described by the time varying nonlinear differential equations:

$$
\dot{x}_{e}=A_{e}(t, \delta, \omega) x_{e}+B_{e}(t, \delta) u_{e}(\delta)
$$

where

$$
\begin{aligned}
A_{e}= & \left(\begin{array}{ccc}
\hat{L}^{-1}(\omega \hat{N}-\hat{R}) & -\hat{L}^{-1}\left(\begin{array}{c}
I_{3} \\
0_{4 \times 3}
\end{array}\right) & 0_{7 \times 1} \\
C_{r}^{-1}\left(I_{3}\right. & \left.0_{3 \times 4}\right) & \left(\begin{array}{ccc}
0 & 0 & 0 \\
0 & 0 & -\omega \\
0 & \omega & 0
\end{array}\right) \\
0_{1 \times 7} & -C_{r}^{-1} P\left(\begin{array}{l}
1 \\
0 \\
L_{r}^{-1}(100) Q
\end{array}\right) & 0
\end{array}\right) \\
B_{e} & =\left(\begin{array}{cc}
-\hat{L}^{-1} & 0_{7 \times 4} \\
0_{4 \times 7} & 0_{4 \times 4}
\end{array}\right)\left(\begin{array}{c}
0_{1 \times 3} \\
I_{3} \\
0_{7 \times 3}
\end{array}\right)
\end{aligned}
$$$$
\hat{L}=\left(\begin{array}{ccccccc}
L_{0} & 0 & 0 & 0 & 0 & 0 & 0 \\
0 & L_{d} & 0 & L_{A D} & L_{A D} & 0 & 0 \\
0 & 0 & L_{q} & 0 & 0 & L_{A Q} & L_{A Q} \\
0 & L_{A D} & 0 & L_{F} & L_{A D} & 0 & 0 \\
0 & L_{A D} & 0 & L_{A D} & L_{D} & 0 & 0 \\
0 & 0 & L_{A Q} & 0 & 0 & L_{Q} & L_{A Q} \\
0 & 0 & L_{A Q} & 0 & 0 & L_{A Q} & L_{G}
\end{array}\right)
$$$$
+\left(\begin{array}{cc}
P \mathbf{L} Q & 0_{3 \times 4} \\
0_{4 \times 3} & 0_{4 \times 4}
\end{array}\right)
$$$$
\left.\hat{N}=\left(\begin{array}{ccccccc}
0 & 0 & 0 & 0 & 0 & 0 & 0 \\
0 & 0 & -L_{q} & 0 & 0 & -L_{A Q} & -L_{A Q} \\
0 & L_{d} & 0 & L_{A D} & L_{A D} & 0 & 0
\end{array}\right)\right)
$$

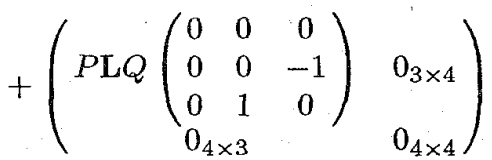$$
\hat{R}=\operatorname{diag}\left\{r_{0}, r_{a}, r_{a}, r_{F}, r_{D}, r_{Q}, r_{G}\right\}+\left(\begin{array}{cc}
P \mathbf{R} Q & 0_{3 \times 4} \\
0_{4 \times 3} & 0_{4 \times 4}
\end{array}\right)
$$

$P=Q^{t}$ is the Park's transformation of [10].
When no thyristors are conducting, the thyristor current $i_{r}=0$, the electrical state vector is $y_{e}=$ $\left(i_{0}, i_{d}, i_{q}, i_{F}, i_{D}, i_{Q}, i_{G}, v_{0}, v_{d}, v_{q}\right)^{t}$, and the dynamics are given by

$$
\dot{y}_{e}=\mathcal{P} A_{e}(t, \delta, \omega) \mathcal{P}^{t} y_{e}+\mathcal{P} B_{e}(t, \delta) u_{e}(\delta)
$$

where $\mathcal{P}$ is the projection matrix $\mathcal{P}=\left(\begin{array}{ll}I_{10} & 0_{10 \times 1}\end{array}\right)$.

The mechanical system state is

$$
x_{m}=\left(\omega_{1}, \omega_{2}, \omega_{3}, \omega_{4}, \omega, \omega_{5}, \theta_{1}, \theta_{2}, \theta_{3}, \theta_{4}, \theta, \theta_{5}\right)^{t}
$$

and the mechanical system differential equations are

$$
\dot{x}_{m}=\left(\begin{array}{cc}
0_{6 \times 6} & \frac{1}{2} H^{-1} K \\
I_{6} & 0_{6 \times 6}
\end{array}\right) x_{m}+\frac{1}{2}\left(\begin{array}{c}
T_{1} / H_{1} \\
T_{2} / H_{2} \\
T_{3} / H_{3} \\
T_{4} / H_{4} \\
-T_{e} / H_{5} \\
0_{7 \times 1}
\end{array}\right)
$$

where $H$ contains the inertia constants, $K$ contains the torsional spring constants and

$$
\begin{aligned}
T_{e}= & \frac{1}{3}\left[\left(L_{d} i_{d}+L_{A D} i_{D}+L_{A D} i_{F}\right) i_{d}\right. \\
& \left.-\left(L_{q} i_{q}+L_{A Q} i_{Q}+L_{A Q} i_{G}\right) i_{q}\right]
\end{aligned}
$$

is the generator torque.

Finally, the generator rotor angle $\theta$ is linked to the power angle by $\theta=\omega_{R} t+\pi / 2+\delta$ where $\omega_{R}$ is the rated angular generator speed; differentiation yields

$$
\dot{\delta}=\omega_{R}-\omega
$$

We now describe the system operation when the combined electro-mechanical system has a steady state periodic solution with period $T=2 \pi / \omega_{R}$. To facilitate understanding, consider Fig. 2.

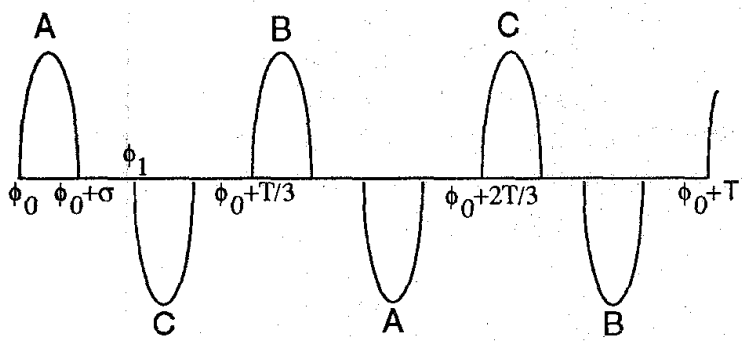

Figure 2. Three phase thyristor currents in one period.

At $\phi_{0}$, the phase $A$ thyristor turns on. This mode, as described by (4.1), (4.3) and (4.5) ends when the thyristor current goes through zero at time $\phi_{0}+\sigma$, and the thyristor is switched off. The non conduction mode, described by (4.2), (4.3) and (4.5), follows the conduction mode and ends when a new firing pulse is applied at $\phi_{1}$ and the phase $C$ thyristor turns on. This starts a new series of similar on and off cycles continuing until time $\phi_{0}+T$, when the next period starts.

The electrical state at a switch on time $\sigma_{\text {on }}$ is denoted by either $y_{e}\left(\sigma_{o n}\right)$ or $x_{e}\left(\sigma_{o n}\right)$, and

$$
x_{e}\left(\sigma_{\text {on }}\right)=\mathcal{P}^{t} y_{e}\left(\sigma_{\text {on }}\right)
$$


Similarly the electrical state at a switch off time $\sigma_{\text {off }}$ is described either by $x_{e}\left(\sigma_{\mathrm{off}}\right)$ or $y_{e}\left(\sigma_{\mathrm{off}}\right)$, and

$$
y_{e}\left(\sigma_{\text {off }}\right)=\mathcal{P} x_{e}\left(\sigma_{\text {off }}\right)
$$

i.e., the matrix $\mathcal{P}$ projects the vector $x_{e}$ onto the plane of zero thyristor current.

Let the electrical and mechanical states be combined to form either a state $x=\left(x_{e}, x_{m}\right)$ when a thyristor is conducting or a state $y=\left(y_{e}, x_{m}\right)$ when all thyristors are off, and let the electrical and mechanical inputs be combined to denote a system input $u=\left(u_{e}, u_{m}\right)$. Then, using (4.1), (4.3), (4.4) and (4.5) and their analogs when a phase $\mathrm{B}$ or $\mathrm{C}$ thyristor conducts, the combined electromechanical system dynamics may be described when a thyristor is conducting as

$$
\dot{x}=A_{\mathrm{on}}(t, \delta, \omega) x+B_{\mathrm{on}}(t, \delta) u(\delta)
$$

and when all thyristors are off as

$$
\dot{y}=A_{\text {off }}(t, \delta, \omega) y+B_{\text {off }}(t, \delta) u(\delta)
$$

where $A_{\text {off }}, B_{\text {off }}$ can be determined from (4.2), (4.3), (4.4) and (4.5) and their analogs when a phase $B$ or $C$ thyristor conducts. At a thyristor switch on, the states are related by (4.6) and at a thyristor switch off, the states are related by (4.7). Equations (4.8) and (4.9) are the time dependent nonlinear differential equations of the combined electromechanical system. The system includes additional nonlinearity because the time of transition from (4.8) to (4.9) is the thyristor switch off time $\sigma_{\text {off }}$ which depends on the system state.

\section{Stability of the periodic steady state}

The dynamics of any system which is periodic with period $T$ can be studied by using the Poincaré map $F$ from dynamical systems theory [8] that advances the system states $x$ forward in time from $\phi_{0}$ to $\phi_{0}+T$, i.e., $F\left(x\left(\phi_{0}\right)\right)=x\left(\phi_{0}+T\right)$. If $F\left(x\left(\phi_{0}\right)\right)=x\left(\phi_{0}\right)$, the system has a steady state passing through $x\left(\phi_{0}\right)$ at time $\phi_{0}$. The Poincaré map can be obtained by integrating the system equations and taking into account the changes in equations and coordinates when the switchings occur.

The small signal stability of the system is computed from the Jacobian $J$ of the Poincare map evaluated at a sampled steady states $x_{0}$, i.e., $J=D F\left(x_{0}\right)$. The eigenvalues of $J$ are the $z$-plane poles of the sampled data transfer function of the linearized system. Our eigenvalue results are presented as continuous time $s$-plane poles $-\alpha+j \beta$ which correspond to the $z$-plane poles $e^{-\alpha T} e^{j \beta T} .-\alpha$ specifies the damping and $\beta$ denotes the frequency of the pole, determined with attention to possible aliasing.

We compute $J$ as follows [8, section 1.5]: First linearize the system equations around the steady state solution. Then $J$ is the linear map that advances a perturbation in state at time $\phi_{0}$, through the linearized system, to $\phi_{0}+T$.
We first linearize the system equations (4.6-4.9) over the interval $\left[\phi_{0}, \phi_{1}\right]$ (see Fig. 2). Equation (4.8) is linearized by linearizing (4.1), (4.3), (4.4) and (4.5). Let $\Delta x_{e}$ be a small perturbation around the steady state periodic solution. Linearizing (4.1):

$$
\begin{aligned}
\Delta \dot{x}_{e}= & A_{e *} \Delta x_{e}+\left(\left.\frac{\partial A_{e}}{\partial \omega}\right|_{*} \Delta \omega+\left.\frac{\partial A_{e}}{\partial \delta}\right|_{*} \Delta \delta\right) x_{e *} \\
& +\left.\frac{\partial B_{e}}{\partial \delta}\right|_{*} u_{e *} \Delta \delta+\left.B_{e *} \frac{\partial u_{e}}{\partial \delta}\right|_{*} \Delta \delta
\end{aligned}
$$

where the subscript $*$ signifies that the expression is evaluated at the steady state. Linearizing (4.5) yields $\Delta \delta=\Delta \theta$, so that we can henceforth replace $\Delta \theta$ by $\Delta \delta$ in the linearized mechanical system state vector $\Delta x_{m}$. Linearizing (4.3) and (4.4) yields

$$
\Delta \dot{x}_{m}=\left(\begin{array}{cc}
0_{6 \times 6} & \frac{1}{2} H^{-1} K \\
I_{6} & 0_{6 \times 6}
\end{array}\right) \Delta x_{m}-\frac{1}{2 H_{5}}\left(\begin{array}{c}
0_{4 \times 1} \\
\left.\frac{\partial T_{e}}{\partial x_{e}}\right|_{*} \Delta x_{e} \\
0_{7 \times 1}
\end{array}\right)
$$

Hence the linearization of (4.8) can be written as

$$
\left(\begin{array}{c}
\Delta x_{e} \\
\Delta \dot{x}_{m}
\end{array}\right)=\left.\left(\begin{array}{cc}
A_{e} & A_{1} \\
A_{2} & A_{m}
\end{array}\right)\right|_{*}\left(\begin{array}{c}
\Delta x_{e} \\
\Delta x_{m}
\end{array}\right)
$$

Carrying out a similar linearization of (4.9) (i.e., (4.2), (4.3), (4.4) and (4.5)) yields

$$
\left(\begin{array}{c}
\Delta \dot{y}_{e} \\
\Delta \dot{x}_{m}
\end{array}\right)=\left.\left(\begin{array}{cc}
\mathcal{P} A_{e} \mathcal{P}^{t} & A_{3} \\
A_{4} & A_{m}
\end{array}\right)\right|_{*}\left(\begin{array}{c}
\Delta y_{e} \\
\Delta x_{m}
\end{array}\right)
$$

where $A_{3}, A_{4}$ are defined similarly to $A_{1}, A_{2}$.

Next we linearize (4.6) and (4.7). Let a thyristor turn on at $s_{\text {on }}$ and note that $s_{\text {on }}$ is a fixed quantity. Linearizing (4.6) yields

$$
\Delta x_{e}\left(s_{\circ \mathrm{on}}\right)=\mathcal{P}^{t} \Delta y_{e}\left(s_{\mathrm{on}}\right)
$$

Switching does not affect the mechanical states and the corresponding relation for the system state is

$$
\Delta x\left(s_{\mathrm{on}}\right)=\mathcal{G}^{t} \Delta y\left(s_{\mathrm{on}}\right)
$$

where $\mathcal{G}^{t}=\left(\begin{array}{cc}\mathcal{P}^{t} & 0 \\ 0 & I_{6}\end{array}\right)$.

If the system is not at a switching time bifurcation $[12,14,15]$, we can linearize (4.7) to get

$$
\Delta y_{e}\left(s_{\text {off }}\right)=\left(\mathcal{P} \dot{x}_{e *}\left(s_{\text {off }}\right)-\dot{y}_{e *}\left(s_{\text {off }}\right)\right) \Delta s_{\text {off }}+\mathcal{P} \Delta x_{e}\left(s_{\text {off }}\right)
$$

But the first term vanishes because (4.7) is satisfied:

$$
\begin{aligned}
\mathcal{P}_{\dot{x}_{e *}}\left(s_{\mathrm{off}}\right)-\dot{y}_{e *}\left(s_{\mathrm{off}}\right)= & \mathcal{P} A_{e *} \dot{x}_{e *}\left(s_{\mathrm{off}}\right)+\mathcal{P} B_{e *} u_{e *}- \\
& \mathcal{P} A_{e *} \mathcal{P}^{t} y_{e *}\left(s_{\mathrm{off}}\right)-\mathcal{P} B_{e *} u_{e *}=0
\end{aligned}
$$

Hence,

$$
\Delta y_{e}\left(s_{\text {off }}\right)=\mathcal{P} \Delta x_{e}\left(s_{\text {off }}\right)
$$

and including the mechanical state as well, we get

$$
\Delta y\left(s_{\text {off }}\right)=\left(\begin{array}{cc}
\mathcal{P} & 0 \\
0 & I_{6}
\end{array}\right) \Delta x\left(s_{\text {off }}\right)=\mathcal{G} \Delta x\left(s_{\text {off }}\right)
$$


This useful simplification is analogous to the simplification in [15] and was overlooked in [13]. The linearization of the system in $\left[\phi_{0}, \phi_{1}\right]$ is thus given by the time varying linear equations (5.3)-(5.6); the linearization in the remaining time intervals can be obtained similarly.

Now we assemble the formula for $J$ by computing the map that advances a small state perturbation $\Delta y\left(\phi_{0}\right)$ from $\phi_{0}$ to $\phi_{0}+T$ through the linearized system. Let $\Phi_{\mathrm{on}}\left(t_{0}, t_{1}\right)$ and $\Phi_{\text {off }}\left(t_{0}, t_{1}\right)$ be the state transition matrices of the linearization of $(4.8)$, (4.9) respectively in the time interval $\left[t_{0}, t_{1}\right]$. Then, the map from $\phi_{0}$ to $\phi_{1}$ through the linearized system is $J_{1}=$ $\Phi_{\text {off }}\left(\phi_{0}+\sigma, \phi_{1}\right) \mathcal{G} \Phi_{\text {on }}\left(\phi_{0}, \phi_{0}+\sigma\right) \mathcal{G}^{t}$, Similarly, the map advancing a perturbation at $\phi_{1}$ to $\phi_{0}+T / 3$ is $J_{2}=$ $\Phi_{\text {off }}\left(\phi_{1}+\sigma, \phi_{0}+T / 3\right) \mathcal{G} \Phi_{\text {on }}\left(\phi_{1}, \phi_{1}+\sigma\right) \mathcal{G}^{t}$, Thus, the map advancing a perturbation in the first subinterval from $\phi_{0}$ to $\phi_{0}+T / 3$ is $J_{2} J_{1}$.

The system states have a three phase symmetry, so the map that advances a perturbation through the linearized system in each subinterval $\left[\phi_{0}+T / 3, \phi_{0}+2 T / 3\right]$, $\left[\phi_{0}+2 T / 3, \phi_{0}+T\right]$ must be the same; hence the Jacobian $J=\left(J_{2} J_{1}\right)^{3}$.

\section{Computational Issues}

The system has 23 states when a thyristor is conducting, and 22 states when all thyristors are off. Newton's algorithm is used to find the periodic steady state solution by solving $F\left(x\left(\phi_{0}\right)\right)=x\left(\phi_{0}\right) . \quad F$ is evaluated by solving the stiff, time varying nonlinear differential equations $(4.8),(4.9)$ using the implicit trapezoidal rule. (The backward Euler method is used for the first few time steps after each switching to avoid numerical oscillations.) An approximate steady state solution to initialize the Newton method is found by approximating the thyristor controlled switched capacitor as an average impedance $[3,4]$ and by using the techniques of [9].

To compute the Jacobian, the state transition matrices $\Phi_{\text {off }}$ and $\Phi_{\text {on }}$ of the linearization of $(4.8),(4.9)$ are found by numerical integration. That is, the fundamental solutions of these linearizations are computed.

\section{Illustrative Eigenvalue Results}

This section shows the results of applying eigenvalue analysis to compute the damping of torsional modes as the thyristor conduction time $\sigma$ varies from $0^{\circ}$ to $48^{\circ}$. The case $\sigma=0^{\circ}$ corresponds to blocking the thyristors. At $\sigma=0^{\circ}$, the TCSC effective reactance $X_{c}$ is $X_{c 0}=-j 0.20$ p.u.. Fig. 3 shows the damping of the modes TM0 through TM5 when the series compensation is $29 \%$. (Negative damping of a mode implies instability of that mode.) The electrical modes are always well damped and are not shown. The mode TM4 has severe negative damping of $-0.45 \mathrm{~s}^{-1}$ when $\sigma=0$, but its damping increases as $\sigma$ increases. The damping of modes TM1, TM2, and TM3 slightly decreases when $\sigma$ is initially increased. The mode TM5 has small damping that is unaffected by $\sigma$ and its graph lies under the zero damp- ing line of Fig. 3. All the torsional modes TM1-TM5 become positively damped for $\sigma>35^{\circ}\left(X_{c}=1.1 X_{c o}\right)$. The frequencies of the torsional modes TM1-TM5 are approximately constant, remaining within $0.5 \mathrm{~Hz}$ of their natural frequencies as $\sigma$ is varied while the TM0 mode has frequency less than $1.4 \mathrm{~Hz}$. The TM0 mode becomes unstable near $\sigma=47^{\circ}$.

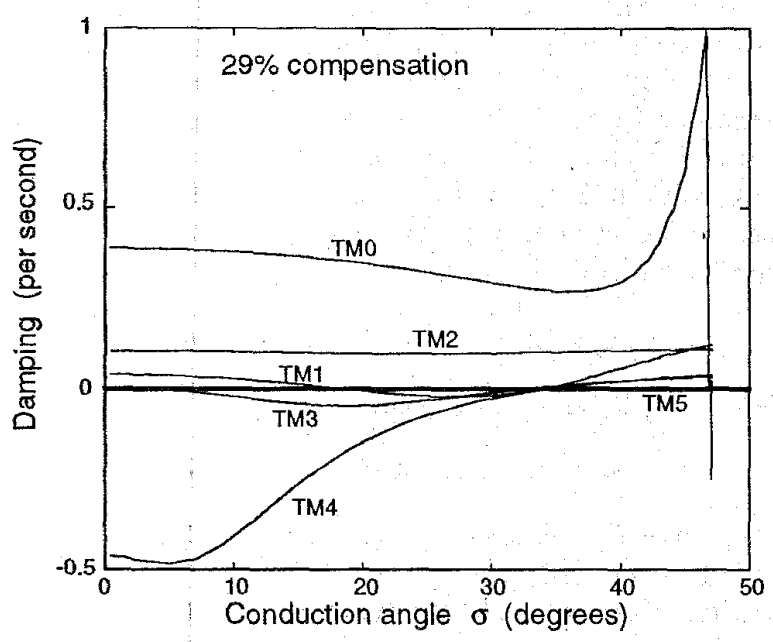

Figure 3. Modal Dampings.

When $\sigma$ is between $35^{\circ}$ and $47^{\circ}\left(X_{c}\right.$ between $1.1 X_{c 0}$ and $1.4 X_{c 0}$ ) the results show positive damping for all modes and hence small signal stability for the system.

For each value of $\sigma$, the solution time for computing the modal dampings is roughly six minutes on an HPRISC machine using a general math package [16].

\section{EMTP Results}

The EMTP is used to simulate the test system to support the eigenvalue results. A detailed machine model, thyristor models with firing synchronized to the line current zeros and an open loop $\sigma$ controller are used. Three cases are considered:

Case 1 illustrates the TCSC damping SSR oscillations when operated at a constant firing angle. The thyristors are blocked at time 0 and a simultaneous threephase line to ground bus fault is simulated at point : A of Fig. 1 at approximately $117 \mathrm{~ms}$ and cleared after $4 \frac{1}{2}$ cycles. (The fault is modeled as parallel impedances of 14.8 and $j 0.04 \mathrm{pu}$.) At $2 \mathrm{~s}$, the thyristors are commanded to operate with $\sigma=40^{\circ}$. Fig. 4 shows a representative torsional response of the shaft. All torques are expressed in per unit deviation from steady state (the base torque is that required to deliver the generator rated mechanical power at synchronous speed). During the first 2 seconds the oscillations associated with the TM4 mode grow. After 2 seconds, the oscillations are damped out by the TCSC operating at $\sigma=40^{\circ}$. The shaft torque of Fig. 4 damps with a time constant of approximately $30 \mathrm{~s}$, i.e., modal damping approximately equal to $0.033 \mathrm{~s}^{-1}$ which 
compares with the computed damping of $0.04 \mathrm{~s}^{-1}$ (in Fig. 3) for the dominant TM4 mode.

Case 2 shows the system instability when $\sigma$ is changed from $40^{\circ}$ to $10^{\circ}$. Initially, the TCSC operates in steady state at $40^{\circ}$. At $1 \mathrm{~s}$, a change in $\sigma$ to $10^{\circ}$ is commanded by ramping $\sigma$ down from $40^{\circ}$ to $10^{\circ}$ in 750 $\mathrm{ms}$. The shaft torsional response of Fig. 5 shows the initial TMO mode decaying and the TM4 mode increasing. The shaft torque of Fig. 5 increases with modal damping approximately equal to $-0.35 \mathrm{~s}^{-1}$ which compares with the computed damping of $-0.41 \mathrm{~s}^{-1}$ (in Fig. 3) for the dominant TM4 mode. The positive and negative modal dampings as $\sigma$ varies in cases 1 and 2 are evident in the EMTP results and are consistent with the behavior predicted by the eigenvalue analysis in Fig. 3.

Case 3 shows the system instability when $\sigma$ is changed from $40^{\circ}$ to $54^{\circ}$. Initially, the TCSC operates in steady state of $\sigma=40^{\circ}$, and a change in $\sigma$ to $54^{\circ}$ is commanded at $1 \mathrm{~s}$ by ramping $\sigma$ up from $40^{\circ}$ to $54^{\circ}$ in 750 ms. Fig. 6 shows the increasing oscillations associated with the low frequency TM0 mode. There is only qualitative agreement between the eigenvalue results and the EMTP when the TM0 mode is unstable. We attribute this to the fact that the equal distance firing assumed for the eigenvalue results is a poor approximation to the synchronization scheme used in the EMTP results when the TMO mode is unstable (the zeros of line current have significant variations when the TM0 mode is unstable). There are indications in the literature suggesting that this instability can be solved by suitable firing angle controls [6].

\section{Conclusions}

We show how to extend the eigenvalue analysis commonly used to study SSR to circuits containing thyristors. In particular, we present eigenvalue analysis for the IEEE first benchmark SSR model with a TCSC. The analysis takes account of time variations and nonlinearities due to the generator and the thyristor switchings. Illustrative eigenvalue results are supported by EMTP simulations.

The eigenvalue results for the first benchmark model show that the thyristor switchings can have a significant effect on system stability and that the effect varies with the firing angle. For simplicity, the firing angle was assumed constant. A study devoted to applying the methods developed in this paper would additionally represent synchronization and control effects by augmenting the equations of Sections 4,5 with those of [11]. Our eigenvalue results are consistent with the damping effect associated with thyristors turning off described in $[12,17]$. Other damping effects involving thyristors are described in $[18,19]$.

The paper contributes an accurate method to compute the damping of SSR modes with a TCSC.

\section{Acknowledgments}

The authors thank Dr. Abdel-Aty Edris for his helpful commerits. The authors gratefully acknowledge funding in part from EPRI under contracts RP 4000-29, RP 8050-03 and RP 8050-04 and from NSF PYI grant ECS-9157192.

\section{References}

[1] IEEE Committee Report, Terms, definitions, and symbols for subsynchronous resonance oscillations. IEEE Trans. on Power Apparatus and Systems, vol. PAS-104, June 1985, pp. 1326-1334.

[2] IEEE Committee Report, Reader's guide to subsynchronous resonance. IIEEE Trans. on Power Systems, vol. 7, no. 1, Feb. 1992, pp. 150-157.

[3] N. Christl et al., Power system studies and modeling for the Kayenta $230 \mathrm{KV}$ substation advanced series compensation. IEE Intl. Conference on thyristor and variable static equipment for AC and DC transmission, London, 1991.

[4] N. Christl, R. Hedin et al., Advanced series compensation (ASC) with thyristor controlled impedance. Cigré 14/37/3805, Paris, Aug.-Sept, 1992.

[5] S. Nyati et al, Effectiveness of thyristor controlled series capacitor in enhancing power system dynamics: an analog simulator study, IEEE Trans. on Power Delivery, vol. 9, no. 2, April 1994, pp. 1018-1027.

[6] E. Larsen, C. Bowler, B. Damsky, S. Nilsson, Benefits of thyristor controlled series compensation. Cigré Paper 14/37/38-04, Paris 1992.

[7] IEEE Committee Report, First benchmark model for computer simulation of subsynchronous resonance, IEEE Trans. on Power Apparatus and Systems, vol. PAS-96, no. 5, Sept. 1977, pp. 1565-1572.

[8] J. Guckenheimer, P. Holmes, Nonlinear oscillations, dynamical systems and bifurcations of vector fields, Springer-Verlag, NY, 1986.

[9] P.M.Anderson,A.A.Fouad,Power system control and stabilityvol. 1, Iowa State Univ. Press, IA, 1977.

[10] P.M. Anderson, B.L. Agrawal, J.E. Van Ness, Subsynchronous resonance in power systems, IEEE Press, NY,1990.

[11] S.G. Jalali, R.H. Lasseter, I. Dobson, Dynamic response of a thyristor controlled switched capacitor, IEEE Trans. on Power Delivery, vol. 9, no 3, July 1994, pp. 1609-1615.

[12] S.G. Jalali, Harmonics and instabilities in thyristor based switching circuits, Ph.D. Thesis, April 1993, University of Wisconsin at Madison.

[13] M. Steinbuch, O.H. Bosgra, Dynamic modeling of a generator/rectifier system IEEE Trans. on Power Electronics, vol. 7, no. 1, Jan. 1992, pp. 212-223.

[14] S.G. Jalali, I. Dobson, R.H. Lasseter, Instabilities due to bifurcation of switching times in a thyristor controlled reactor. Power Electronics Specialists Conference, Toledo, Spain, July 1992, pp. 546-552.

[15] I. Dobson, S.G. Jalali, Surprising simplification of the Jacobian of a diode switching circuit. IEEE Intl. Symposium on Circuits and Systems, Chicago, IL, May 1993, pp. 2652-2655.

[16] The MathWorks, Inc., MATLAB User's Guide, Natick, MA, 
1993.

[17] I. Dobson, S.G. Jalali, R. Rajaraman, Damping and resonance in a high power switching circuit, in Systems and Control Theory for Power Systems (eds. J.H. Chow, P.V. Kokotovic, R.J. Thomas), IMA volume 64 in mathematics and its applications, Springer Verlag, 1994, pp. 137-156.

[18] N.G. Hingorani, A new scheme for subsynchronous resonance damping of torsional oscillations and transient torque - Part I. IEEE PES Summer Meeting, Minneapolis, MN, 1980.

[19] R.A. Hedin, K.B. Stump, N.G. Hingorani, A new scheme for subsynchronous resonance damping of torsional ascillations and transient torque-Part II, IEEE PES Summer Meeting, Minn., MN, 1980.

\begin{tabular}{llllllllll}
\multicolumn{8}{c}{ Appendix. System parameters } \\
\begin{tabular}{lllllllll}
$L_{0}$ & $L_{d}$ & $L_{A D}$ & $L_{F}$ & $L_{D}$ & $L_{q}$ & $L_{A Q}$ & $L_{G}$ & $L_{Q}$ \\
\hline 0.135 & 1.79 & 1.66 & 1.722 & 1.665 & 1.71 & 1.58 & 1.906 & 1.675 \\
$r_{0}$ & $r_{F}$ & & $r_{D}$ & $r_{G}$ & $r_{Q}$ \\
\hline 100 & $0.53 / 377$ & $1.54 / 377$ & $5.3 / 377$ & $3.1 / 377$ \\
$r_{a}=r_{b}=r_{c}=0, \quad C_{r}=1 / 0.2$, & $L_{\tau}=\frac{1}{7 C_{r}}$, & $\omega_{R}=1$
\end{tabular}
\end{tabular}

$L$ has elements 1.0533 on the diagonal and 0.3533 off the diagonal. $\mathrm{R}$ has elements 0.18 on the diagonal and 0.16 off the diagonal. Mechanical dampings (in 1/sec) for the modes TM1-TM5 [7] are:

\begin{tabular}{lllll} 
TM1 & TM2 & TM3 & TM4 & TM5 \\
\hline 0.05 & 0.11 & 0.028 & 0.028 & 0
\end{tabular}

The generator operating conditions are power output $=0.9$, power factor $=0.9$, and terminal voltage $=1.0$.

Rajesh Rajaraman (S'92) received the B. Tech and MS in Electrical Engineering from IIT, Bombay, India and the University of Maine-Orono. He is completing his $\mathrm{PhD}$ at the University of Wisconsin-Madison. His interests include subsynchronous resonance, utility power electronics and voltage collapse.

Ian Dobson (M'89) received the $\mathrm{PhD}$ in Electrical Engineering from Cornell University and joined the University of WisconsinMadison faculty in 1989. His current interests are bifurcations, nonlinear dynamics, voltage collapse and switching circuits.

Robert H. Lasseter (F'92) received the PhD degree in Physics at the University of Pennsylvania, Philadelphia in 1971. He was a Consultant Engineer at General Electric Company until he joined the University of Wisconsin-Madison in 1980. His main interest is the application of power electronics to utility systems.

Yihchih Shern (S'92) received the BS in Electrical Engineering from the University of Rochester and the MS in General Engineering from the University of Illinois at Urbana-Champaign. He is completing his MS at the University of Wisconsin-Madison. His main interest is utility power electronics.

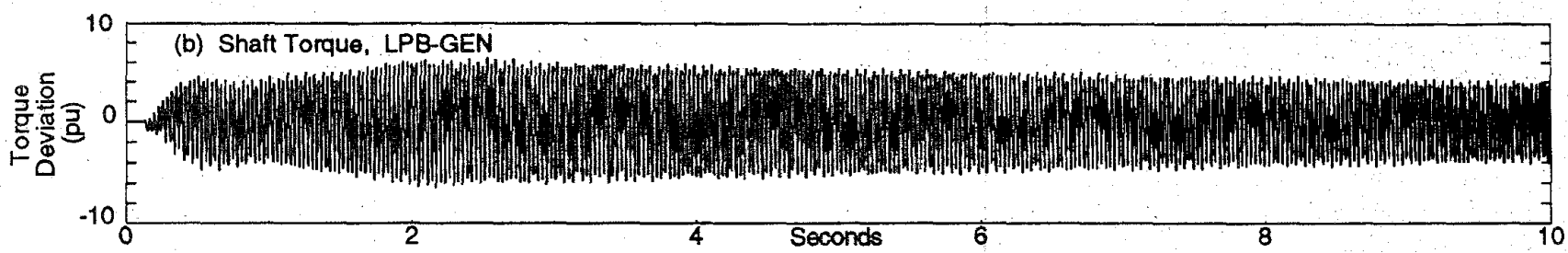

Figure 4. Response curves for case 1. $\left(\sigma=0^{\circ}-->40^{\circ}\right)$

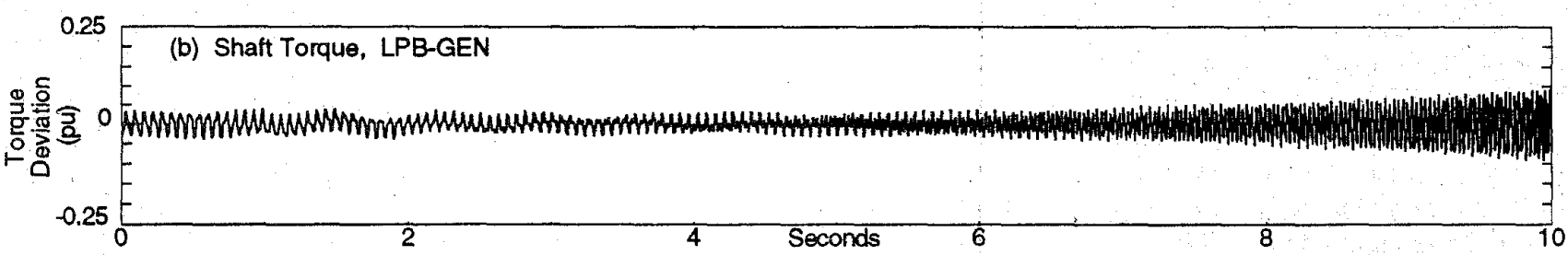

Figure 5. Response curves for case 2. $\left(\sigma=40^{\circ} \ldots>10^{\circ}\right)$

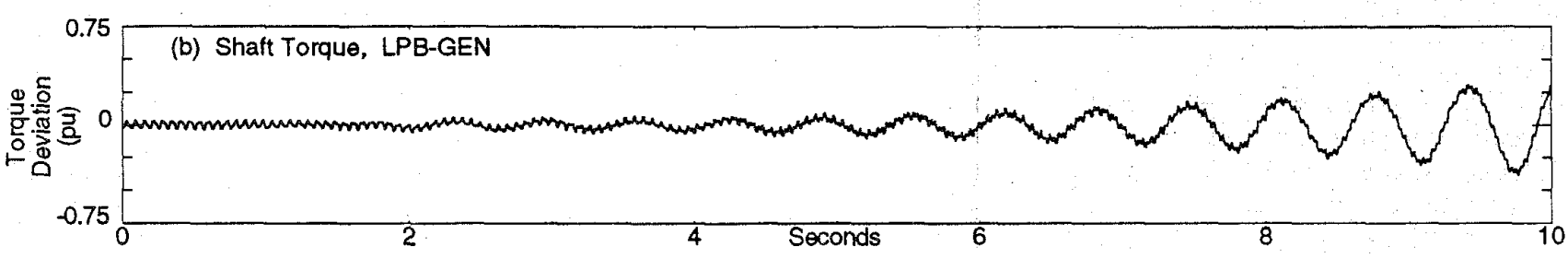

Figure 6. Response curves for case $3 .\left(\sigma=40^{\circ}-->54^{\circ}\right)$ 


\section{Discussion}

SASAN G. JALALI and RON A. HEDIN

Siemens Energy and Automation

Atlanta, GA :

The authors are to be congratulated for an excellent work on extending the eigenvalue analysis in [11] to study SSR for systems including TCSC. The method is accurate and its results can be used for benchmarking other models.

The equations (4.1) and (4.2) use Parks transformation to describe the dynamics of the IEEE first benchmark with TCSC. These equations are time varying, in contrast to the time invariant equations which describe the dynamics of the IEEE first benchmark with only conventional capacitors.

As is indicated in the paper, the time varying nature of the system equations impose difficulties in computing the Poincare map and its Jacobian. In short, the map needs to be numerically evaluated by integrating a set of nonlinear and time varying equations. The Jacobian is the product of the state transition matrices of the linearized system equations which are also time varying and thus need to be evaluated along the periodic trajectory.

The difficulties in numerically computing the Jacobian makes the suggested eigenvalue analysis impractical for large power systems with one or more TCSC. We would appreciate the authors point of view on this comment.

Manuscript received August 28, 1995.

Rajesh Rajaraman, Ian Dobson, Robert H. Lasseter: We thank Sasan Jalali and Ron Hedin for their comments. They correctly summarize the computations necessary when the equations are time varying to evaluate the Jacobian and hence determine the system stability from the eigenvalues.

When applied to more complex TCSC models, there is also effort required to take account of the dependence of TCSC synchronization on previous events such as current zero crossings in the computation of the Jacobian. We have found some iteration necessary to solve for this dependence in three phase power systems (this aspect will be described in a forthcoming EPRI report). When applied to larger power systems, there is the computational burden mentioned by the discussers. While our approach applies to the general case, at some size or complexity, the approach will become burdensome. We would be more optimistic than the discussers about the feasibility of the computations for small power systems with approximately 3 generators and 2 TCSC devices. It should be noted that the method is much less burdensome than detailed EMTP simulations.

This does raise the question of approximate methods which are sufficiently accurate and easy. Torque per unit velocity methods are well established as an excellent approximate method when there is no TCSC and the Park transformed equations are independent of time. In recent work we have also generalized the torque per unit velocity methods to the time varying case and applied them to the first benchmark model with a TCSC $[A 1, A 2]$. These results show a good match with the exact eigenvalue methods of this paper.

Thus we recommend using the eigenvalue method of the paper for small power systems and generalized torque per unit velocity methods for larger power systems. It is important to note that since the eigenvalue method of the paper is exact, it can be used as a benchmark to validate other approximate methods and to verify small signal models of the TCSC.

We take this opportunity to correct misprints in equation (4.4), which should read

$$
\begin{aligned}
T_{e}=\frac{1}{3} & {\left[\left(L_{d} i_{d}+L_{A D} i_{D}+L_{A D} i_{F}\right) i_{q}\right.} \\
& \left.-\left(L_{q} i_{q}+L_{A Q} i_{Q}+L_{A Q} i_{G}\right) i_{d}\right]
\end{aligned}
$$

References

[A1] R. Rajaraman, Damping of subsynchronous resonance and nonlinear dynamics in thyristor switching circuits, PhD thesis, University of Wisconsin, Madison, WI 53706, 1995.

[A2] R.Rajaraman, I.Dobson, Damping estimåtes of subsynchronous and power swing oscillations in power systems with thyristor switching devices, Report ECE 95-9, Electrical and Computer Engineering Dept., University of Wisconsin, Madison, WI 53706, July 1995.

Manuscript received October 18, 1995. 